\title{
Caracterização de "Caminhos mais prováveis" em uma rede complexa de processos jurídicos
}

\author{
J. L. B. de Araújo ${ }^{1}$, João A. Monteiro Neto ${ }^{4}$, F. S. de Siqueira ${ }^{1}$, \\ C. H. Moura Santos ${ }^{1}$,R. G. de Vasconcelos ${ }^{1}$, \\ Erneson A. Oliveira ${ }^{2,3}$, Carlos Caminha ${ }^{2}$,Vasco Furtado ${ }^{2}$ \\ ${ }^{1}$ Laboratório de Ciência de Dados e Inteligência Artificial - Universidade de Fortaleza (UNIFOR) \\ 60.811-905 - Fortaleza - CE - Brazil \\ ${ }^{2}$ Programa de Pós Graduação em Informática Aplicada - Universidade de Fortaleza (UNIFOR) \\ 60.811-905 - Fortaleza - CE - Brazil \\ ${ }^{3}$ Mestrado Profissional em Ciências da Cidade - Universidade de Fortaleza (UNIFOR) \\ 60.811-905 - Fortaleza - CE - Brazil \\ ${ }^{4}$ Curso de Direito - Universidade de Fortaleza (UNIFOR) \\ 60.811-905 - Fortaleza - CE - Brazil \\ jlbdearaujo@gmail.com, joaoneto@unifor.br,fsiqueira@edu.unifor.br \\ clausmoura@edu.unifor.br,ricardo.vasconcelosetjce.jus.br \\ \{erneson, caminha, vasco\}eunifor.br
}

\begin{abstract}
A judicial process is essentially formed by a sequence of acts that are translated into procedural changes that carry the history of the development of the activities carried out by the most diverse parties in the course of the procedural demand. In this article, we explain the most likely paths that characterize the classes of legal proceedings through a set of rules attributed to a complex network. We investigated the sequence of activities most likely and highlight the average times required to complete. We observed what would be the main points of delays in the possible ways of starting and ending a process in addition to the similarity between the possible paths taken. In addition, we indicate how much time would be spent, on average, in increasing the number of movements for each class of process. The characterization of these networks and the contextualization of the ideal movement path, associated with the other data generated by the proposed method, allow, through a reliable data generation process, a detailed observation of the flow of lawsuits, the identification of atypical behaviors and the planning of interventions capable of optimizing the provision of the judicial service by the judiciary.
\end{abstract}

Resumo. Um processo judicial é formado, em essência, por uma sequência de atos que são traduzidos em movimentações processuais que carregam o histórico do desenvolvimento das atividades realizadas pelas mais diversas partes no curso da demanda processual. Neste trabalho, explicitamos os caminhos mais prováveis que caracterizam as classes de processos judiciais por meio de um conjunto de regras atribuídas a uma rede complexa. Observamos a sequência de atividades mais prováveis e destacamos os tempos médios 
necessários para serem concluídas. Observamos quais seriam os principais pontos de atrasos nas possíveis formas de inicialização e término de um processo além da similaridade entre os possíveis caminhos tomados. Ademais, indicamos qual seria o tempo gasto, em média, no aumento do número de movimentações para cada classe de processo. A caracterização dessas redes e a contextualização do caminho de movimentação ideal, associado aos outros dados gerados pelo método proposto permitem, por meio de um processo confiável de geração de dados, uma observação detalhada do fluxo dos processos judiciais, a identificação de comportamentos atípicos e o planejamento de intervenções capazes de otimizar a prestação do serviço jurisdicional pelo judiciário.

\section{Introdução}

Um processo judicial tem por finalidade eliminar conflitos de interesses divergentes fazendo uso justo das leis aplicáveis às matérias discutidas no curso da relação processual. De modo geral, um processo judicial engloba uma série de etapas, denominadas de movimentações processuais, delimitadas e orientadas pelos marcos legais e temporais do processo, e são realizados por todos os atores envolvidos na relação processual, Juiz, Promotor de Justiça, Advogados, Partes e Serventuários do Judiciário. Estruturados de forma lógica, mas não linear, essas movimentações guiam o desenvolvimento do processo desde o seu início até a sua conclusão. Etapas como Petição inicial, Citação, Réplica, Sentença e Recursos são movimentações comuns na maioria dos processos. Em essência, elas carregam informações, na forma sequencial, tais como identificação de quem ajuizou a ação, verificação das leis exigidas, argumentos de defesa do réu, apresentação de provas e a decisão final. Tais movimentações observadas dependem e variam significativamente dependendo das matérias envolvidas (direito criminal, direito de família, etc.) o que torna ainda mais complexo obter padronização sequencial entre os processos jurídicos. Apesar da existência de uma padronização base tendo em conta o tipo de processo, o conjunto de movimentações não dependem exclusivamente das classes judiciais as quais os processos são atribuídos inicialmente, sendo muitas vezes influenciada pela 'história' do processo e das necessidades que o Juiz pode ter de realizar os mais diversos atos que possam fundamentar sua decisão final, o que cria dessa forma novas movimentações e ciclos de ações necessárias para que o mesmo seja finalizado que são divergentes do caminho inicialmente previsto.

Geralmente, o acréscimo no número de movimentações dentro de um processo faz com que o mesmo demore mais tempo a ser finalizado. Assim, a visualização de um "caminho processual"ótimo ou sub-ótimo torna-se de grande interesse tanto para as partes envolvidas no caso como particularmente para o Poder Judiciário que pode avaliar o nível de eficiência de suas unidades identificando possíveis desvios e projetando intervenções para correção de incongruências identificadas [Koniaris et al. 2017]. Mesmo que esses caminhos possam ser demasiadamente complicados de serem observados matematicamente, além das complexidades de ações subjetivas tomadas pelas partes e pelo juiz no curso de um processo, observar quais seriam as movimentações que acarretam os maiores atrasos ou maiores acelerações pode servir como um importante elemento encorajador para ações pontuais de maximização dos serviços prestados pelo poder judiciário.

Dessa forma, devido a dificuldade de se estabelecer uma sequência padrão 
de movimentações processuais, apresenta-se aqui uma metodologia alternativa de caracterização dos processos por meio de interações em redes complexas [Newman 2003, Barabási and Bonabeau 2003, Wang and Chen 2003]. Na seção (2) será apresentado uma visão geral do problema proposto. Na seção (3) serão apresentados as regras matemáticas de criação das redes complexas para as diversas classes processuais escolhidas. Apresentamos também os caminhos mais prováveis baseados nos dados oriundos das Tabelas Processuais do Tribunal de Justiça (TJ). Os tempos gastos para cada etapa e as conexões entre os caminhos mais importantes são destacados durante o texto. Por fim, condensamos os resultados na seção (4) onde destacamos uma maneira eficiente de obter a influência dos acréscimos possíveis de tempo devido a necessidade de novas movimentações. Na seção (5) destacamos os principais pontos da nossa pesquisa com as principais perspectivas.

\section{Estado da arte}

A teoria das redes complexas e uma área altamente interdisciplinar que oferece recursos para o estudo dos mais variados tipos de sistemas complexos. Muitos processos dinâmicos não-lineares e simulações estocásticas podem ser analisados em redes complexas, tais como: as interações entre as proteínas, relacionamento humano, sistemas de transporte aéreo, a Internet, o mercado financeiro, dentre outros sistemas complexos

[Boccaletti et al. 2006]. É importante comentar que tais matérias não são necessariamente excludentes. Geralmente, fenômenos complexos carregam informações de interações entre múltiplas variáveis onde as influências dos atributos especulados dentro do conteúdo estudado relevam, por partes, às relações de multiescala podendo assim, quando possível, observar a ascendência de um agente microscópico dentro da rede complexa ao qual seus efeitos geram grandes impactos dentro do aglomerado macroscópico analisado. Tipicamente, estudos voltados à interações humanas (especialmente dentro de regiões urbanas), seja por meio de comunicação remota ou através de contatos diretos em redes de mobilidade, produzem resultados tanto de interesse econômico como político. Efeitos secundários de tais interações podem ser vista em redes de transportes urbanos, proliferação de doenças, disseminação de informação, entre outros. Dessa forma, encontrar gargalos de informações, distinguir pontos de máximo poder de difusão de informação, verificar possíveis desperdícios e lucros, são possíveis produtos oriundos de análises através de redes complexas [Caminha et al. 2017, Caminha 2012].

A análise de rede complexa é uma ferramenta crítica para a compreensão de vários sistemas complexos [Amaral and Ottino 2004], inclusive como uma abordagem para obter uma compreensão quantitativa da estrutura e evolução do direito [Ruhl et al. 2017]. Cientistas da ciência da computação e especialistas jurídicos têm usado métodos de análise de citações, a fim de construir redes de citações de jurisprudência, bem como para modelar e quantificar a complexidade do corpus legislativo [Lupu and Voeten 2012].

Tem sido também utilizada para analisar dados de redes complexas construídas a partir de decisões de tribunais nacionais e internacionais, de estatutos, de constituições e tratados internacionais. Há também trabalhos neste contexto que exploram, por exemplo, quais características de sistemas complexos ocorrem no direito estatutário, como as referências à decisões judiciais são usadas para moldar argumentos jurídicos, ou onde a dinâmica social existe entre juízes ou árbitros internacionais. Chandler [J. 2005] examinou a estrutura de rede complexa de decisão judicial baseada em precedentes, usando dados da Suprema Corte dos Estados Unidos. Além disso, a partir de estudos voltados 
para criação de índices de qualidades de advogados do Rio de Janeiro, tornou-se possível analisar os perfis dos mesmos em relação à atividades voltadas para os processos jurídicos trabalhistas. Métricas matemáticas comumente utilizadas em estudos de redes complexas como closeness, betweenness, força do vértice e pagerank tornaram-se essenciais para ranquear a eficiência de diversos advogados apresentando sua importância na comunidade em que trabalha [Ribeiro et al. 2016].

Apesar de existirem inúmeros trabalhos que caracterizam e analisam dados em redes complexas, não foi encontrado qualquer referência que estudasse as movimentações dos processos jurídicos, em busca de identificar e apresentar os caminhos mais prováveis de processos judiciais por meio de um conjunto de regras atribuídas a uma rede complexa.

Portanto, uma das contribuições deste trabalho é partir dos dados das classes e movimentações dos processos judiciais da justiça estadual cearense, representá-los como uma rede complexa, modelando as relações existentes de tal forma que se torne possível a caracterização dos processos judiciais.

\section{Metodologia}

Os dados utilizados foram baseados nas classes de movimentações das Tabelas Processuais Unificadas do Poder Judiciário criadas a partir da Resolução do CNJ no 46/2007, bem como no registro de movimentações internas operadas no âmbito da Justiça Estadual cearense. Foram considerados os processos de $1^{\circ}$ grau protocolados até o ano de 2019 e baixados em 2019 nas diversas instâncias do Tribunal de Justiça do Estado do Ceará, nas classes: "7 - Procedimento Comum Cível", "1116 - Execução Fiscal", "436 - Procedimento do Juizado Especial Cível"e "159 - Execução de Título Extrajudicial". No total foram analisadas a quantidade de 4797 processos para a classe Execução de Título Extrajudicial, 67499 para Procedimento Comum Cível, 29643 Procedimento do Juizado Especial Cível e 23039 para Execução Fiscal com pelo menos 300 tipos de movimentações diversas.

A nossa base de dados oriundas do TJ contêm múltiplas informações que dão características para os processos. Destacamos que para esse trabalho foram usados dados das classes judiciais nomeadas como Execução de Título, Procedimento Comum Cível, Procedimento do Juizado Especial Cível e Execução Fiscal como sendo as classes mais demandadas da Justiça Estadual avaliada. As informações temporais para cada movimentação apresentada revelam suas inicializações. Assim, a cada movimentação iniciada, indicando uma série de atividades ao qual a compete, uma outra etapa termina, indicando que o termo "movimentação"está associado a um estado temporal de um processo. Nesse caso, rotulamos como $\Delta T$ os tempos duradouros de cada movimentação dentro de um processo. Identificamos em cada etapa do processo qual é o par $O L D \rightarrow N O W$ como sendo a construção de uma junção entre o tipo de movimentação em uma sequência anterior $(O L D)$ e a sequência atual $(N O W)$. Dessa forma, é possível montar uma tabela de dados contendo todos os fluxos entre tipos de movimentações consecutivas, além de obter os dados dos tempos gastos entre tais movimentações. Assim, definimos como $F_{i j} \mathrm{O}$ número de sequências observadas que surgiram inicialmente do estado $i$ e estão no estado $j$.

A matriz $\mathbf{F}$ não é simétrica visto que é possível obter elemento com $F_{i j} \neq F_{j i}$. Dessa forma, a assimetria do problema sugere a construção espacial de uma rede de ações 
direcionadas onde os nós carregam as informações de cada tipo de movimentação e as arestas vinculam às evoluções sequenciais sintonizadas por $F_{i j}$. Na Figura (1) é apresentada uma ilustração da formação de criação da rede complexa processual. Os pontos indicam cada tipo de movimentação e as setas indicam os vetores de transformação entre movimentações sequenciais.
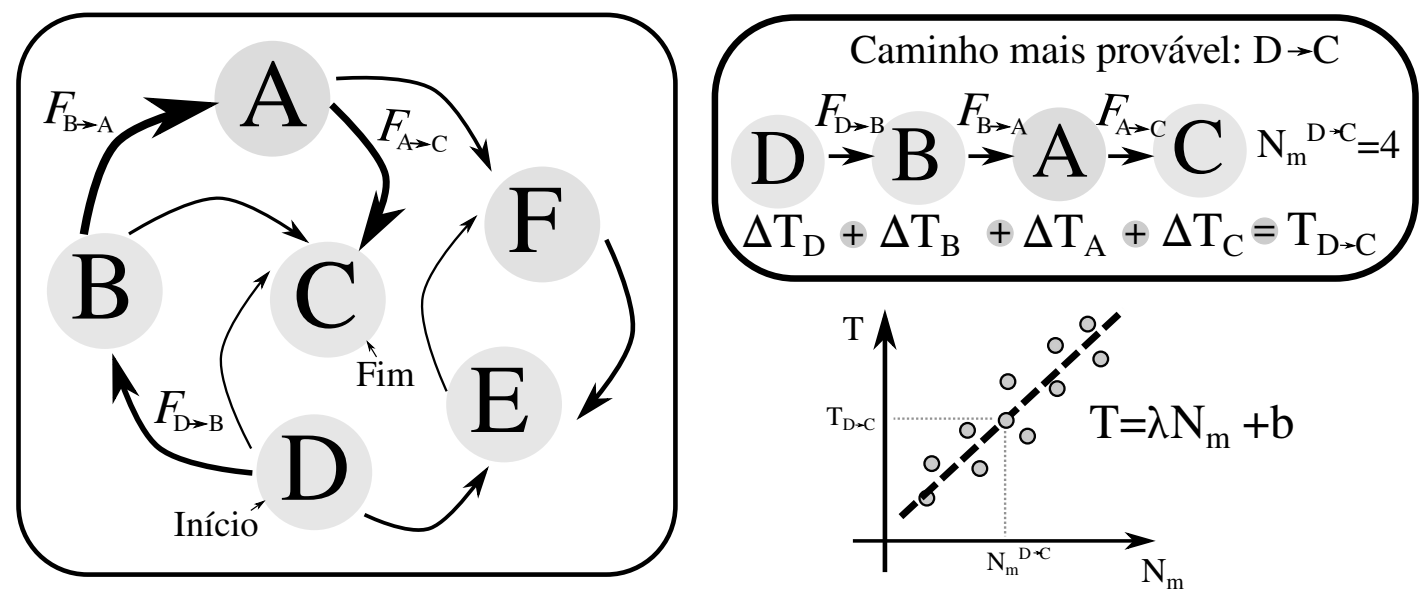

Figura 1. À esquerda é apresentado um modelo esquemático imaginário de uma rede complexa direcional para tipos de movimentações contidas em uma classe judicial. Cada nó da rede contém um tipo de movimentação (ilustrados por A, B, C, D, E e F). As diversas larguras das setas representam os pesos associados dessa rede aos quais são regulados pelos índices $F_{i j}$. À direita, uma sequência $D \rightarrow B \rightarrow A \rightarrow C$ é apresentada indicando o caminho mais provável entre os processos jurídicos que inicializam em $D$ e terminam em $C$. Neste exemplo, a sequência de caminho mais provável possui $N_{M m}^{D \rightarrow C}=4$ movimentações com um tempo total $T_{D \rightarrow C}$. Assim, a partir de todas as possíveis combinações entre movimentações de inícialização e término de um processo jurídico, é possível obter uma relação linear entre $T$ e $N_{m}$ gerando um coeficiente angular $\lambda$ que reflete, em média, um acréscimo de tempo à um processo jurídico para cada movimentação adicional.

Dessa forma, pretende-se, para cada tipo de classe judicial, construir caminhos entre as movimentações que represente-a como sendo construída, em sua essência, pelas movimentações que serão visitadas.

Uma maneira indicada de criar o caminho mais provável é verificar qual seria o tipo de movimentação mais frequente para a primeira etapa de cada classe judicial. A partir daí, os próximos pontos seriam acessados pela incidência de $F$ criando assim um caminho ótimo local. Esse tipo de estratégia é visto em técnicas de Greedy algorithm. Porém, em sistemas de elevadas ramificações e tomadas de decisão com probabilidades entre movimentações similares, essa abordagem pode induzir a um grande número de sequências de elevadas degenerescências ou produzir caminhos sub-ótimas temporais. Uma alternativa é verificar entre todas as combinações possíveis de caminhos qual seria aquele com menor custo, ou mais probabilidade de acontecer. Para esse fim, criamos definimos uma matriz $D^{e f}$, chamada de distância efetiva, ao qual seus elementos são regulados por: 


$$
D_{i j}^{e f}=\exp \left(-\beta F_{i j}\right)
$$

onde $\beta=\frac{1}{\left\langle F_{i j}\right\rangle}$ regula a influência de $F_{i j}$. Dessa forma, dizemos que o caminho mais provável (CP) de um processo é encontrado minimização de todas as rotas $D_{i j}^{e f}$, ou seja:

$$
C P_{i j}=\operatorname{Dijkstra}\left(D_{i j}^{e f}\right)
$$

onde Dijkstra [Dijkstra et al. 1959] é a função que retorna os pontos da redes com menor custo regulado por $D_{i j}^{e f}$. Observa-se na Figura (1) a ilustração de um percusso otimizado obtido quando se inicializa um processo jurídico pela movimentação $D$ e o termina na $C$. Nesse contexto, o processo tem $N_{m}=4$ movimentações na sequência $D \rightarrow B \rightarrow A \rightarrow C$ com um custo temporal $T$. É importante mencionar que as escolhas de $D$ e $C$ foram totalmente arbitrárias e que o caminho mais provável é balanceado pelos termos de mais intensidade de $\mathbf{F}$. Porém, um conjunto de resultados oriundos de todas as combinações possíveis entre movimentações de inicialização e movimentações de término podem nos mostrar um qualitativo da classe processual estudada. Nesta etapa, destacamos um parâmetro $\lambda$ que caracteriza os efeitos temporais médios do acréscimo de uma ou mais movimentações processuais para uma dada classe analisada. Dessa forma, nosso trabalho consiste em etapas que capturam dados de processos judiciais de uma dada classe jurídica, transforma-os em uma matriz de sequência de movimentações $\mathbf{F}$ e encontra todos os CP's produzindo assim um parâmetro $\lambda$ que qualifica a classe jurídica. Além disso, é possível observar os pormenores para cada sequência observada dando enfase às combinações de inicialização e término de um processo em ordem de maior frequência. Com isto é possível verificar o tempo médio gasto durante os pares de movimentações mais influentes da classe jurídica provendo uma maior clareza ao analisar as movimentações.

\section{Resultados}

As movimentações processuais possuem nomes e significado diversos. As ações internas que as compõem esclarecem suas atividades criando assim um fluxograma de tarefas que ora depende de outras movimentações anteriores à elas dentro de uma processo jurídico, ora nutri a produção diversas outras movimentações posteriores às mesmas. A complexidade entre "causas e efeitos"tornasse cada mais confusa diante de fatos internos do processo que dependerá tanto dos atores envolvidos como tema discutido no processo. Assim, é importante comentar que mesmo que o nome de uma movimentação seja autoexplicativa, poderão existir pormenores a esta que tratem de assuntos completamente diferentes. Um exemplo simples é uma movimentação nomeada como Documento. Á principio indica a construção, ou verificação, ou até a requisição de documentos pelas partes envolvidas no processo. Para entender qual ação foi tomada é necessário a compreensão dos textos produzidos durante o processo. Nosso trabalho não constituiu em tratar dados nesse nível. Porém, por meio da equação (2) tornasse possível destacar as movimentações mais relevantes dentro de um processo e, através de uma análise mais minuciosa, verificar os motivos das dispersões temporais dentro de movimentação. 
Dessa forma, para cada classe judicial, obtivemos as movimentações que caracteriza o caminho de etapas mais prováveis $(\mathrm{CP})$. Na Figura (2) apresentamos um painel que representa o tempo médio gasto em cada tipo de movimentação para as diversas classes estudadas. Para essa etapa destacamos como sendo pontos relevantes de construção do caminhos aqueles pares que movimentações $F_{i j}$ mais comuns para inicializar e terminar um processo. Assim, para a classe Execução de Título Extrajudicial (Figura (2(a))) o caminho mais provável indica ações que levam às movimentações: Protocolo de Petição $\rightarrow$ Em classificação $\rightarrow$ Processo apto a ser distribuído $\rightarrow$ Distribuição por sorteio $\rightarrow$ Autuação $\rightarrow$ Concluso ao juiz $\rightarrow$ Recebimento $\rightarrow$ Concluso para Despacho $\rightarrow$ Mero expediente $\rightarrow$ Encaminhamento edital/relação para publicação $\rightarrow$ Despacho/Decisão disponibilizado no Diário de Justiça Eletrônico $\rightarrow$ Trânsito em julgado $\rightarrow$ Definitivo. Nota-se que durante a movimentação do tipo Recebimento houve o maior gasto de tempo. Nas classes Procedimento Comum Cível, Procedimento do Juizado Especial Cível e Execução Fiscal (Figuras (2(b)-(d))) os tipos de movimentações preferências são diversos. Para a classe Procedimento Comum Cível observamos a sequência Conclusão $\rightarrow$ Mero expediente $\rightarrow$ Encaminhado edital/relação para publicação $\rightarrow$ Despacho/Decisão disponibilizado no Diário de Justiça Eletrônico $\rightarrow$ Trânsito em julgado $\rightarrow$ Definitivo $\rightarrow$ Expedição de Certidão de Arquivamento com destaque temporal, representado acentuações em atrasos, nas movimentações Mero expediente e Trânsito em julgado. Similarmente é possível observar nas classes Procedimento do Juizado Especial Cível e Execução Fiscal onde as movimentações nomeadas como Expedição de Carta e Certidão emitida criam atrasos de grandes tempos.

É importante mencionar que as quantidades de movimentações listadas no caminho mais provável de cada classe de processo depende da escolha de seus marcos de inicialização e fechamento. Estatisticamente, os pares mais influência se destacaram dos demais. Além disso, é possível que existam caminhos com elevado grau de similaridade devido às movimentações comuns, mesmo em rotas de mais ou menos etapas.

Assim, para cada par possível de inicialização e fechamento de um processo, dentro de uma determinada classe, calculamos as variáveis $N_{m}$ e

$$
T=\sum_{k} \Delta T_{k}
$$

que são, respectivamente, o número de movimentações encontradas para se obter o caminho mais provável entre os nós da rede e o tempo total gasto por esse caminho para cada etapa imersa nele que demorou um tempo $\Delta T$. Por exemplo, como foi apresentado na Figura (2(a)), escolhidos como pontos de partidas e fechamento, respectivamente, as movimentações Protocolo de Petição e Definitivo, temos uma quantidade de $N_{m}=13$ movimentações e um total de $T=257$ dias. Na Figura (3) são apresentados os gráficos de dispersão entre tais variáveis. Nota-se, novamente, que a escala de tempo e a quantidade de movimentações exigidas para o obter o caminho mais provável depende da escolha da classe estudada. É importante comentar que a relação entre as variavéis sugerem uma correlação linear na forma $T=\lambda N_{m}+b$ [Press et al. 1992] onde $\lambda$ indica, em média, o acrescimo de tempo atribuido a um processo a uma variação unitária ao número de movimentações daquela classe especifica. As retas em destaque na Figura (3) indicam o ajuste linear entre as variáveis com coeficiente de Pearson [Freedman et al. 2007] maior 

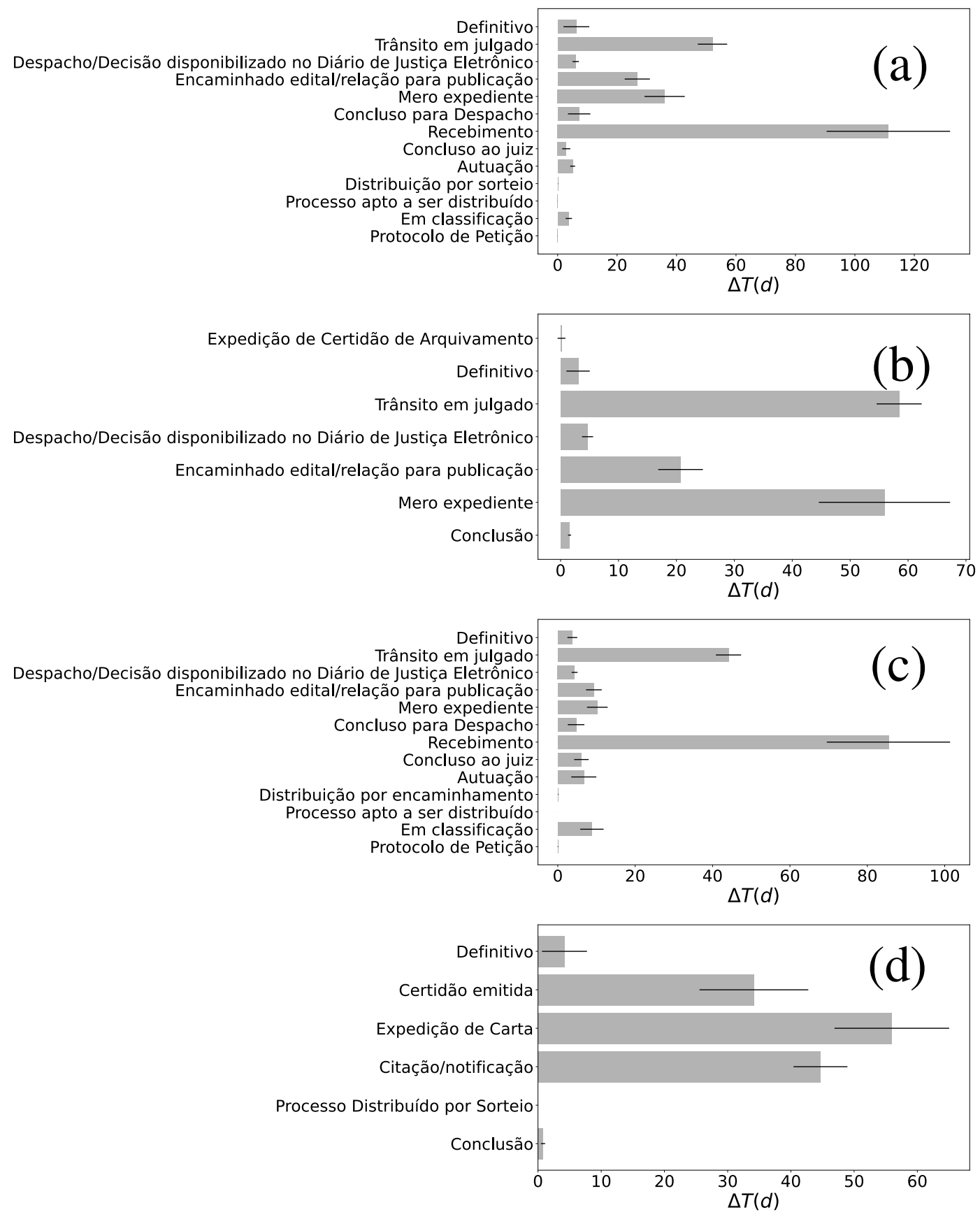

Figura 2. Tempos médios gastos $(\Delta T)$, em dias, para diversas movimentações nas classes (a) Execução de Título Extrajudicial , (b) Procedimento Comum Cível, (c) Procedimento do Juizado Especial Cível e (d) Execução Fiscal. Linhas horizontais indicam o desvio padrão de $\Delta T$. Os nós de inicialização e fechamento de um processo são indicados na parte vertical de cada gráfico na sua forma ascendente. Por exemplo, em (a), um processo inicia com a movimentação do tipo Protocolo de Petição e termina com a movimentação Definitivo.

que 0.72 para qualquer tipo de classe citada.

Os valores de $\lambda$ obtidos pelo ajuste linear para as classes Execução de Título 

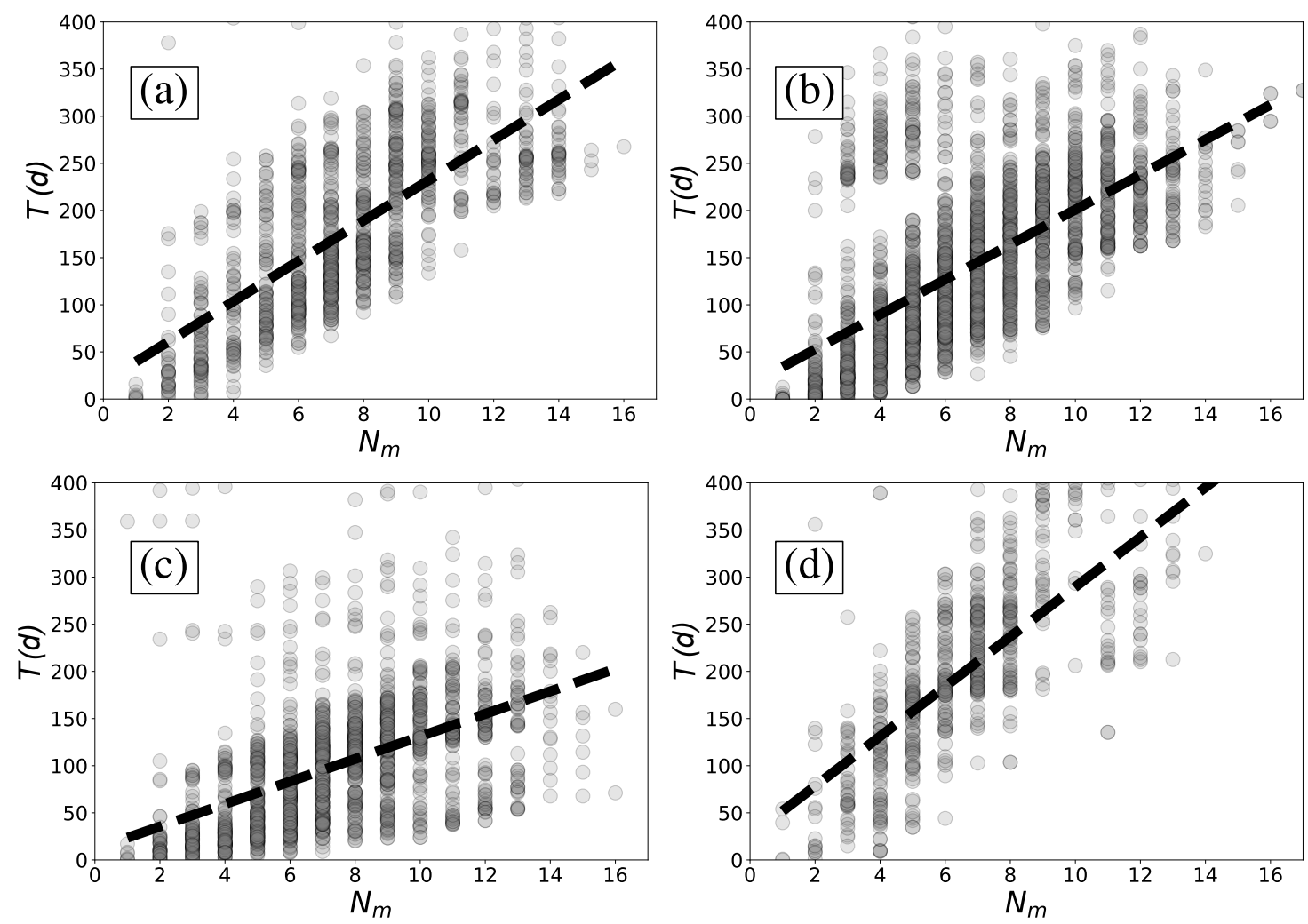

Figura 3. Tempo total gasto $T$, em dias, por um processo obtidos por caminho mais provável. Cada ponto indica uma combinação de uma possível movimentação de inicialização com outra possível movimentação de fechamento gerando uma sequência de $N_{m}$ movimentações totais. Os resultados referem-se as classes (a) Execução de Título Extrajudicial , (b) Procedimento Comum Cível, (c) Procedimento do Juizado Especial Cível e (d) Execução Fiscal, respectivamente. As retas em destaques referem-se aos ajustes lineares na forma $T=\lambda N_{m}+b$.

Extrajudicial, Procedimento Comum Cível, Procedimento do Juizado Especial Cível e Execução Fiscal, são, respectivamente, 21.3 dia/movimentação, 11.5 dia/movimentação, $11.9 \mathrm{dia} /$ movimentação e $26.4 \mathrm{dia} /$ movimentação. Seus valores refletem, graficamente, a inclinação da reta nos gráficos da Figura (3) levando em consideração as dispersões de pontos no gráfico. Assim, para cada classe, é possível estimar os efeitos de atrasos causados pelo aumento do número de movimentações e ranquear aquelas classes que possuem maior velocidade no fechamento de um processo jurídico.

\section{Conclusão}

O trabalho desenvolvido contribui para o desenvolvimento de pesquisas nas área de construção e avaliação de redes no contexto jurídico, ao mesmo tempo que possui potencial para permitir produção de estruturas datificadas de fácil extração capazes de orientar a implantação de ações de correção e política públicas direcionadas ao aumento de produtividade e melhor prestação do provimento de justiça.

A metodologia utilizada para a criação e avaliação dos dados gerados pelas movimentações processuais configura-se como inovadora por dois importantes fatores. O primeiro é que utiliza uma abordagem combinando análise de dados e construção de 
redes ao contexto legal, abordagem com pouca referência na literatura da computação como na literatura jurídica ou de engenharia jurídica. O segundo é porque observa e valoriza uma fonte de informação normalmente menosprezada e pouco valorizada nos estudos dedicados a entender a caracterização, dinâmica e eficiência de processos judiciais.

Por fim, o conjunto de dados gerados pela caracterização das redes movimentação processual, especialmente as inferências geradas pela visualização dos intervalos de tempo entre as movimentações e também da predição de duração dos processos, ante o fluxo de movimentações que eles tomam, permite aos gestores do poder judiciário acesso a dados importantes para tanto comparar níveis de performance entre distintas unidades jurisdicionais como também verificar a ocorrência de 'gargalos' de eficiência. Um bom exemplo desse potencial é a observação de que no processo 159 - Execução de Título Extrajudicial'(Figura (2(d))), a simples expedição de uma carta demora em média mais de 50 dias, o que indica a necessidade de adoção de medidas corretivas para que a prestação jurisdicional seja mais rápida e eficiente.

\section{Referências}

Amaral, L. and Ottino, J. (2004). Complex networks. augmenting the framework for the study of complex systems. European Physical Journal B, 38:147-.

Barabási, A.-L. and Bonabeau, E. (2003). Scale-free networks. Scientific american, 288(5):60-69.

Boccaletti, S., Latora, V., Moreno, Y., Chavez, M., and Hwang, D.-U. (2006). Complex networks: Structure and dynamics. Physics Reports, 424(4):175-308.

Caminha, C. (2012). Modeling user reports in crowdmaps as a complex network.

Caminha, C., Furtado, V., Pinheiro, V., and Ponte, C. (2017). Detecção de comunidades em redes complexas para identificar gargalos e desperdício de recursos em sistemas de ônibus. In Anais do VI Brazilian Workshop on Social Network Analysis and Mining, Porto Alegre, RS, Brasil. SBC.

Dijkstra, E. W. et al. (1959). A note on two problems in connexion with graphs. Numerische mathematik, 1(1):269-271.

Freedman, D., Pisani, R., and Purves, R. (2007). Statistics (international student edition). Pisani, R. Purves, 4th edn. WW Norton \& Company, New York.

J., C. S. (2005). The network structure of supreme court jurisprudence. university of houston law center. SSRN, (2005).

Koniaris, M., Anagnostopoulos, I., and Vassiliou, Y. (2017). Network analysis in the legal domain: a complex model for European Union legal sources. Journal of Complex Networks, 6(2):243-268.

Lupu, Y. and Voeten, E. (2012). Precedent in international courts: A network analysis of case citations by the european court of human rights. British journal of political science, 42(2):413-439.

Newman, M. E. (2003). Mixing patterns in networks. Physical review E, 67(2):026126.

Press, W. H., Teukolsky, S. A., Vetterling, W. T., and Flannery, B. P. (1992). Numerical Recipes in C. Cambridge University Press, Cambridge, USA, second edition. 
Ribeiro, L., Figueiredo, D., and Nascimento, P. (2016). Análise e ranqueamento da rede de advogados induzida por processos judiciais trabalhistas. In Anais do V Brazilian Workshop on Social Network Analysis and Mining, pages 13-24, Porto Alegre, RS, Brasil. SBC.

Ruhl, J. B., Katz, D. M., and Bommarito, M. J. (2017). Harnessing legal complexity. Science, 355(6332):1377-1378.

Wang, X. F. and Chen, G. (2003). Complex networks: small-world, scale-free and beyond. IEEE circuits and systems magazine, 3(1):6-20. 